\section{PILOT STUDY EXPLORING THE REGIONAL REPOLARISATION INSTABILITY INDEX IN RELATION TO MYOCARDIAL HETEROGENEITY AND PREDICTION OF VENTRICULAR ARRHYTHMIA AND DEATH}

doi:10.1136/heartjnl-2011-300198.159

${ }^{1} \mathrm{~W}$ B Nicolson, ${ }^{1} \mathrm{C} D$ Steadman, ${ }^{1} \mathrm{P}$ Brown, ${ }^{2} \mathrm{M}$ Jeilan, ${ }^{2} S$ Yusuf, ${ }^{2} S$ Kundu, ${ }^{2} \mathrm{~A}$ J Sandilands, ${ }^{2} \mathrm{P}$ J Stafford, ${ }^{1} \mathrm{~F}$ S Schlindwein, ${ }^{2} \mathrm{G}$ P McCann, ${ }^{1} \mathrm{G}$ A Ng. ${ }^{1}$ University of Leicester, Leicester, UK; ${ }^{2}$ University Hospitals of Leicester NHS Trust, Leicester, UK

Introduction There is a need for better sudden cardiac death (SCD) risk markers. Mounting evidence suggests that the mechanism underlying risk of ventricular arrhythmia (VA) is increased heterogeneity of electrical restitution. We investigated a novel measure of action potential duration (APD) restitution heterogeneity: the Regional Repolarisation Instability Index (R2I2) and correlated it with peri-infarct zone (PIZ) a cardiac magnetic resonance (CMR) anatomic marker of VA risk.

Methods Blinded retrospective study of 30 patients with ischaemic cardiomyopathy assessed for an implantable cardioverter defibrillator. The R2I2 was derived from high resolution 12 lead ECG recorded during programmed electrical stimulation (PES). ECG surrogates were used to plot APD as a function of diastolic interval; the R2I2 was the maximal value of the mean squared residuals of the mean points for anterior, inferior and lateral leads normalised to the mean value for the total population. PIZ was measured from late gadolinium enhanced CMR images using the full width half maximum technique.

Results Seven patients reached the endpoint of VA/death (median follow-up 24 months). R2I2 > median was found to be predictive of $\mathrm{VA} /$ death independent of PES result, left ventricular ejection fraction and ORS duration (6/14 vs $1 / 15 \mathrm{p}=0.031)$. Modest correlation was seen between the R2I2 and PIZ ( $r=0.41 \mathrm{p}=0.057$ ) (Abstract 159 figure 1).

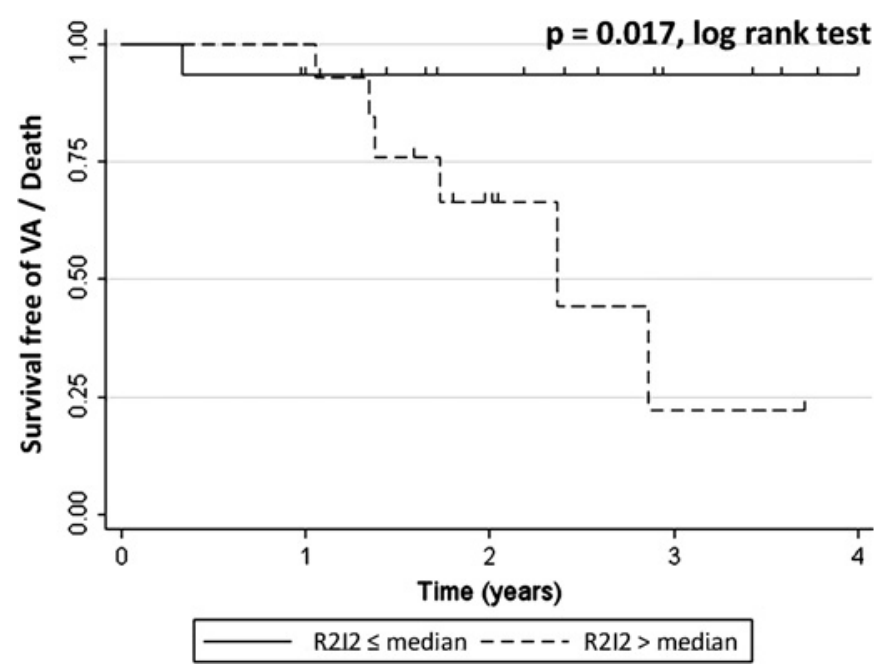

Abstract 159 Figure 1

Conclusions In this pilot study of ischaemic cardiomyopathy patients, the R2I2 was shown to be an electrical measure of VA/ death risk with a moderately strong correlation with an anatomic measure of arrhythmic substrate, the extent of PIZ. The R2I2 may add value to existing markers of VA/death and merits further investigation.
Abstract 159 Table 1

\begin{tabular}{lclcl}
\hline Variable & $\begin{array}{l}\text { Whole Group } \\
(\mathbf{n = 3 0 )}\end{array}$ & $\begin{array}{l}\text { No VA/death } \\
(\mathbf{n = 2 3 )}\end{array}$ & $\begin{array}{l}\text { VA/death } \\
(\mathbf{n = 7})\end{array}$ & $\mathbf{p}$ \\
\hline Age (years) & $67 \pm 9$ & $65 \pm 9$ & $72 \pm 8$ & 0.055 \\
Sex (\% male) & 97 & 96 & 100 & \\
QRSD(ms) & $107 \pm 20$ & $107 \pm 21$ & $106 \pm 15$ & 0.95 \\
EF(\%) & $31 \pm 14$ & $32.4 \pm 15$ & $27 \pm 7.5$ & 0.34 \\
PES result (positive/total) & $12 / 30$ & $7 / 23$ & $5 / 7$ & 0.068 \\
R2I2>median & $14 / 29$ & $8 / 22$ & $6 / 7$ & 0.031 \\
EDV index (ml/cm) & $1.48 \pm 0.41$ & $1.49 \pm 0.41$ & $1.45 \pm 0.45$ & 0.84 \\
SV index (ml/cm) & $0.42 \pm 0.14$ & $0.43 \pm 0.14$ & $0.39 \pm 0.15$ & 0.47 \\
Follow-up (months) & $24(18)$ & $24(16)$ & $16(16)$ & 0.088 \\
PIZ \% & $7.8(10.7)$ & $7.5(8.4)$ & $13.6(8.5)$ & 0.093 \\
Scar \% & $10.9(16.5)$ & $9.67(13.5)$ & $21.9(17.8)$ & 0.16 \\
\hline
\end{tabular}

160 HIGH DOSE OCTREOTIDE; A NOVEL THERAPY FOR THE TREATMENT OF DRUG REFRACTORY POSTURAL ORTHOSTATIC TACHYCARDIA SYNDROME IN PATIENTS WITH JOINT HYPERMOBILITY SYNDROME

doi:10.1136/heartjnl-2011-300198.160

A E French, C Shepherd, A Horne, C Parker, J Tagney, J Pitts-Crick, T Johnson, G Thomas. Bristol Heart Institute, Bristol, UK

Introduction Postural orthostatic tachycardia syndrome (POTS) is defined as symptomatic orthostatic intolerance with an increase in heart rate of 30 beats per minute within 10 min of head up tilt (HUT). This dysautonomia causes wide-ranging symptoms including palpitations, presyncope, chronic fatigue, headache and cognitive difficulties. When POTS occurs in patients with preexisting Joint Hypermobility Syndrome (JHS), symptoms begin approximately a decade earlier than non-JHS patients with a preponderance of neurological features, secondary to cerebral hypoperfusion. Vascular laxity with splanchnic venous pooling has been implicated as a causative factor thus measures to expand plasma volume (thereby increasing mean arterial pressure and restoring cerebral perfusion) form the mainstay of therapy. Symptomatic improvements have been previously reported in POTS patients with the somatostatin analogue Octreotide, a powerful splanchnic vasoconstrictor. We report the first UK series of JHS patients with drug refractory POTS treated with high-dose octreotide.

Methods Six patients (female, aged 21-52) were referred to our institution. All had known JHS (4 requiring a wheelchair), neurological symptoms (headache and cognitive impairment) and diagnostic tilt-table testing with a mean increase in heart rate of 64 beats/min (range 47-73) with head-up tilt (HUT). All patients had remained symptomatic despite pre-treatment with a mean of 5 POTS medications (range 5-7) including fludrocortisone, midodrine, propranolol, ivabradine, selective serotonin reuptake inhibitors, gabapentin and erythropoietin. Octreotide was commenced using a short-acting preparation given 3 times daily (dosage $50-250 \mu \mathrm{g}$ according to body mass) in conjunction with a longacting (monthly), intramuscular injection (dosage 10-30 mg). The short-acting preparation was weaned following the second monthly injection.

Results During follow-up of 3 months (range 1-8), 3 (50\%) patients reported a complete resolution of all postural and neurological symptoms which corresponded with a normalised response to HUT. The remaining patients reported a dramatic improvement but ongoing postural symptoms. No patients developed supine hypertension. Side effects including mild abdominal discomfort and transient diarrhoea were reported in $3(50 \%)$ patients.

Conclusion Octreotide is increasingly recognised as an effective therapy in POTS patients. Both short-acting, subcutaneous $(0.9 \mu \mathrm{g} /$ $\mathrm{Kg})$ and long-acting, intramuscular $(10-20 \mathrm{mg}$ ) preparations have 